\title{
NBS
}

Eechnical Mote

\section{A PEAK AC-DC VOLTAGE COMPARATOR FOR USE IN A STANDARDS LABORATORY}

L. A. MARZETTA

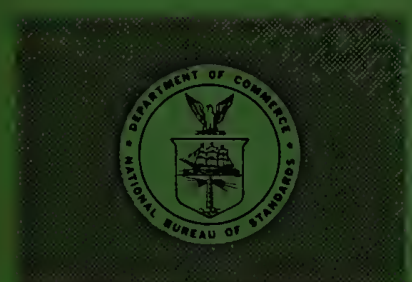

U. S. DEPARTMENT OF COMMERCE NATIONAL BUREAU OF STANDARDS 
THE NATIONAL BUREAU OF STANDARDS

The National Bureau of Standards is a principal focal point in the Federal Government for assuring maximum application of the physical and engineering sciences to the advancement of technology in industry and commerce. Its responsibilities include development and maintenance of the national standards of measurement, and the provisions of means for making measurements consistent with those standards; determination of physical constants and properties of materials; development of methods for testing materials, mechanisms, and structures, and making such tests as may be niecessary, particularly for government agencies; cooperation in the establishment of standard practices for incorporation in codes and specifications; advisory service to government agencies on scientific and technical problems; invention and development of devices to serve special needs of the Governmont; assistance to industry, business, and consumers in the development and acceptance of commercial standards and simplified trade practice recommendations; administration of programs in cooperation with United States business groups and standards organizations for the development of international standards of practice; and maintenance of a clearinghouse for the collection and dissemination of scientific, technical, and engineering information. The scope of the Bureau's activities is suggested in the following listing of its three Institutes and their organizational units. Institute for Basic Standards. Applied Mathematics. Electricity. Metrology. Mechanics. Heat. Atomic Physics. Physical Chemistry. Laboratory Astrophysics." Radiation Physics. Radio Standards Laboratory:* Radio Standards Physics; Radio Standards Engineering. Office of Standard Reference Data.

Institute for Materials Research. Analytical Chemistry. Polymers. Metallurgy. Inorganic Materials. Reactor Radiations. Cryogenics. Materials Evaluation Laboratory. Office of Standard Reference Materials.

Institute for Applied Technology. Building Research. Information Technology. Performance Test Development. Electronic Instrumentation. Textile and Apparel Technology Center. Technical Analysis. Office of Weights and Measures. Office of Engineering Standards. Office of Invention and Innovation. Office of Technical Resources. Clearinghouse for Federal Scientific and Technical Information.*"

*Located at Boulder, Colorado, 80301.

**Located at 5285 Port Royal Road, Springfield, Virginia, 22171. 


\title{
NATIONAL BUREAU OF STANDARDS Technical Note 280
}

\author{
ISSUED JANUARY 17,1966
}

\section{A PEAK AC-DC VOLTAGE COMPARATOR FOR USE IN A STANDARDS LABORATORY}

\author{
L. A. Marzetta \\ Electronic Instrumentation Section \\ Measurement Engineering Division \\ National Bureau of Standards
}

NBS Technical Notes are designed to supplement the Bureau's regular publications program. They provide a means for making available scientific data that are of transient or limited interest. Technical Notes may be listed or referred to in the open literature.

\footnotetext{
For sale by the Superintendent of Documents, Government Printing Office
} Washington, D.C., 20402 - Price 25 cents 
Contents

Page

1. Introduction -.-......-1

2. Study of Peak Voltage Detecting Circuits --...-.--.-- 4

2.1 Use of Diode Rectifier-.........- 4

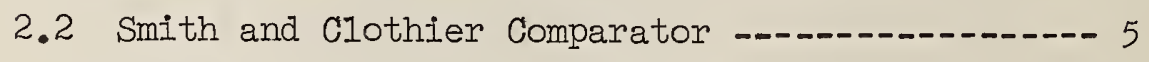

2.3 Resistance Summing Comparator -.---.--.-.-.--- 6

3. Description of NBS Peak ac-de Transfer Standard --..- 7

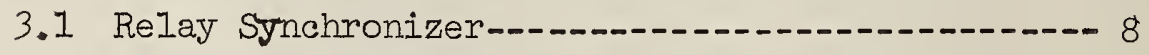

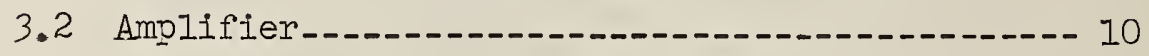

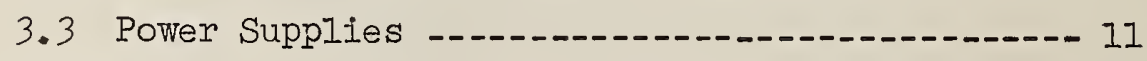

4. Test Results -

Table 1 -

Table 2 -

Table 3 -

Table 4 -

List of Figures -...-..- 19 


\title{
A. Peak ac-dc Voltage Comparator
}

For Use in a Standards Laboratory

\section{Louis A. Marzetta}

\begin{abstract}
An increased availability of high-precision a-c and $d-c$ power sources has allowed routine electrical measurements to be made in the field with a precision previously possible only in the standards laboratory. Ac-dc comparisons are being made on the basis of the peak, rms, and average value of the sine wave source. The peak ac-dc voltage comparator described in this report was developed for use in the standards laboratory to $h \cong l p$ meet the increasing demand for the certification of new field instruments. The described instrument is capable of establishing the ac-dc error of other peak comparators at frequencies from 50 to $2400 \mathrm{~Hz}$ with an imprecision of less than $15 \mathrm{ppm}$.
\end{abstract}

Key words: Voltage, alternating, direct, calibration, comparator, standard, peak and transfer.

\section{Introduction}

A brief look into the past history of electrical measurement could be helpful in understanding the evolution of instrumentation for use in a standards laboratory. The structure for the derivation of electrical units gained a firm foundation with the efforts of Gauss, Weber, and later, Maxwell, who in 1861 headed a program for correlating electrical and mechanical units. In the early use of electricity, such as telegraphy, quantitative measurements involving resistance and direct current received the greatest attention. Later in the 1880's the use of electricity took a heavy turn in the direction of power and lighting. Alternating current electrical energy generation and distribution lagged that of direct current by only about one year. Thus the needs for ac-dc transfer techniques and instruments were established. For the next 60 years or so $d-c$ measurements held a very wide advantage in precision over a-c measurements. Great improvements had come about in resistance measuring bridges. Around the turn of the century Weston perfected an electrochemical cell vich offered the standards laboratory a $d-c$ reference voltage source ith accurate level predictability and long term constancy. 
In 1958, F. Hermach (1) writing on the state of precise a-c measurement stated, "The accuracy of a-c measurement is often limited by the stability of the source rather than the instrument." Until recent years the source of a-c power at audio frequencies in standards laboratories was the rotary alternator. Despite the handicaps with such sources of sine wave voltages, laboratories around the world had achieved high precision rms a-c measurements. At NBS, the work of F. K. Harris (2) and F. B. Silsbee (3) in the 1930's resulted in the perfection of electrodynamic instruments such that ac-dc difference measurements could be made with a precision of $0.01 \%$ at power frequencies and about $0.1 \%$ at frequencies close to $3 \mathrm{kHz}$. The rms value of measurement was a natural selection since the majority of physical effects in experiments and other usage are related to the transformation of electrical energy to heat dissipated in a current carrying resistor or to mechanical forces developed between current carrying conductors or electrically charged plates. Since harmonic contamination affects rms measurement very little, moderate wave form distortion was not very troublesome at that time. Total harmonic distortion of a few percent could be tolerated when making accurate determinations. The precision of measurement with electrodynamic instruments had reached a leveling-off stage, and by nature their frequency response was severely limited.

The next stage in the progress of precise a-c measurement came in the field of electro-thermal ac-dc transfer instruments, especially the contributions by F. Hermach of NBS in the 1940's and $50^{\prime} \mathrm{s}$ (1), (4), (5). His development of the differential thermocouple transfer standard opened the door to measurement precision of better than $0.01 \%$ throughout the audio range of frequencies and extended precise a-c determinations well into the radio frequency spectrum. This work came at a time when it was

(1) F. L. Hermach, "AC-DC Transfer Instruments for Current and Voltage Measurements", IRE Transactions on Instrumentation, pp. 235-240, Dec. 1958.

(2) F. K. Harris, "A Suppressed Zero Electrodynamic Voltmeter", J. Res. N.B.S., Vo1. 3, pp. 445-457, 1929.

(3) F. B. Silsbee, "Composite Coil Electrodynamic Instruments", J. Res. N.B.S. , Vo1. 8, pp. 217-264, 1932.

(4) F. I. Hermach, E. S. Williams, "A Wide-Range Volt-Ampere Converter for Current and Voltage Measurements", AIEE Transactions, pt. 1 (Communications and Electronics), Vol. 78, Sept. 1959, pp. 384-388.

(5) J. E. Griffin and F. L. Hermach, "A Differential Thermocouple Voltmeter", AIEE Transactions, (Communication and Electronics), Vol. 81, No. 63, pp. 339, November 1962. 
needed not only to enrich the capabilities of the many standards laboratories but also to meet the urgent requirements for precise a-c measurements brought on by rapid advances in guidance equipment associated with the defense buildup.

In 1954 W. Smith and W. Clothier (6) of NPL, Australia, reported a measurement study that although it did not attract much attention at that time may now be classed as a pioneering work of significant contribution to a-c measurement. Although the USA, as well as laboratories in other countries, had for years concentrated on electrodynamic transfer instruments, the Australians and the British had placed their faith in an electrostatic voltmeter transfer standard. They believed that by overcoming the surface contamination problems of the capacitor plates, the electrostatic voltmeter could offer ac-dc transfer precision of better than $0.01 \%$, (100 ppm). What is most revealing in the paper is that Smith and Clothier proposed to use a peak-to-peak ac-de voltage comparator (with an electronic amplifier and oscilloscope) as the primary means of $a-c$ to $d-c$ transfer determination, and then to use the values so obtained to establish the ac-dc error difference of the electrostatic instrument. (More details will be given later on this procedure.) In order to make use of peak-to-peak measurement, the authors had to give serious attention to the control of the amplitude stability and waveform purity of the alternating potential source. With a well designed electronic oscillator, feedback amplifier, and harmonic filter, their a-c source had an amplitude stability of $0.001 \%$ per minute and a total harmonic content of $0.001 \%$ at a single frequency of $50 \mathrm{~Hz}$. The overall performance of the system resulted in a measurement precision of $0.001 \%$ to $0.003 \%$.

A number of years elapsed before electronic techniques and devices were used with ac-dc standards. In $1962 \mathrm{P}$. Richman presented one of several papers that appeared on this subject (7) (8). Sparked by demands of the military, Richman and others ushered in the era of "Electronic Standards" with wide range, audio frequency, power standard generators having total harmonic distortion of less than $0.01 \%$, with a similar figure for week to week amplitude stability, and $d-c$ supplies with $0.005 \%$ stability for long periods of operation. Other instruments included peak ac-dc voltage comparators and an average ac-dc transfer standard. The

(6) W. E. Smith and W. K. Clothier, "Determination of the D.C./A.C. Transfer Error of an Electrostatic Voltmeter", The Proceedings of the Institution of Electrical Engineers, Part 2 (Power Engineering), No. 83, pp. 465-469, Oct. 1954.

(7) P. Richman, "A New Peak-to-DC Comparator for Audio Frequencies", IRE Transactions on Instrumentation, Vo1. 1-11, Nos. 3 and 4 , p. 115, Dec. 1962.

(8) P. Richman, "A New Absolute AC Voltage Standard", Paper 13, Session 26, IEEE Record of Convention held March, 1963. 
work described in this report was initiated to help meet the increasing demand for certification of new instruments.

\section{A Study of Peak Voltage Detecting Circuits}

If an instrument is to be developed for the purpose of comparing the peak value of a sine wave with an equivalent $d-c$ value, the instrument must have an electronic circuit that performs some type of signal transformation at the time of the sine wave peak. The following is a brief review of a number of designs that have been considered, including a few statements of their relative merits or shortcomings.

\subsection{Use of a Diode Rectifier as a Peak Voltage Detector}

For a rectifier with almost ideal characteristics, the d-c output voltage of Figure 1 will be close to the peak value of the input sine function for the polarity in the forward conduction direction. Near ideal characteristics in a rectifier imply a forward rectifier resistance that is orders of magnitude lower in value than the load resistance $R_{0}$, and a reverse direction resistance orders higher than $R_{0}$. In addition to this, the $R_{0} C$ time constant must be many times greater than the period of the input waveform, and the rectifier must have no contact potential.

Consideration of the output time constant, $R_{0} C$, is necessary in order to establish the desired degree of accuracy in converting peak a-c to $d-c$. The droop of the $d-c$ output from one sine peak to the next

is an exponential decay of the form $e^{-T / R_{0} C}$. If the load time constant is very much greater than the input period, $T$, the droop is almost linear and the $d-c$ value for half-wave rectification will be in error by one-half of the droop percentage. For a few parts per million accuracy, the load time constant must be several thousand times the input function period; a situation that leaves the overall system with a very slow response time. Slow response coupled with ever present amplitude instability at the source and extraneous noise will lead to serious errors. One compromise would be to reduce the $R_{0} C$ time constant and accept a computable error.

As it turns out, the problem stated is academic since available diodes are far from ideal. A test of the high quality solid state diodes reveals a forward resistance value of $10^{8}$ ohms for a voltage of 3 millivolts; in fact, the forward resistance is still $10^{6}$ ohms at 0.3 volts for a silicon diode. In addition the same diode suffers from back conduction. At about 20 millivolts potential the reverse resistance is only twice the forward resistance. Thermionic diodes have better resistance characteristics but possess a high unstable contact potential of about 0.8 volt. 
Before giving up on the peak rectifier circuit, two modified arrangements should be mentioned. If the diode rectifier in Figure 1 is repleced by a synchronous mechanical switch or by a synchronized ring diode gate, the dilemma of a long charging time constant and short system response time can be resolved. With a synchronous switch, the long output time constant can be kept in the interest of low droop at the output; yet the circuit can retain the advantage of cycle to cycle peak charge restoration. It might be said that the system has both fast response and long term peak memory storage. The price involved for this innovation is a switch that can open and close for the briefest period exactly at the peak of the sine wave; a duration in the vicinity of one-half degree of angle. Some form of impedance translating circuit would have to be used in order to transfer the $\mathrm{d}-\mathrm{c}$ potential at the high resistance $\mathrm{R}_{\mathrm{O}} \mathrm{C}$ network to an output with a low resistance for subsequent measurement of the $d-c$. Possibly a highly stabilized electrometer amplifier could be used.

\subsection{The Smith and Clothier Time Selection} Peak ac-dc Voltage Comparator

Smith and Clothier (6) were probably first in recognizing the potentiality for precision peak measurement with the development of a nearly pure sine wave a-c source. With such a source made available, they designed a peak-to-peak ac-dc voltage transfer device to be used as a standard at an a-c frequency of $50 \mathrm{~Hz}$. The end purpose was to use the peak comparator as a means for determining the ac-de error of an electrostatic voltmeter which was itself used as a working transfer standard.

The principle of their peak-to-peak comparator is shown in Figure 2. A circuit was developed by means of which a high speed relay can be synchronized with the sine wave signal, $V_{1}$, closing its contacts briefly at each alternation peak. Two stable d-c sources, $\mathrm{Vb}_{1}$ and $\mathrm{Vb}_{2}$, serve the purpose of shifting the average position of the sine wave, $V_{1}$, so that each peak when alternately examined by the synchronous relay will have its level positioned near reference ground. The values of $\mathrm{Vb}_{1}$ and $\mathrm{Vb}_{2}$ are adjusted by watching the switching pattern on an oscilloscope. It consists of a sequential positive and negative peak pulse train. Proper adjustment of $\mathrm{Vb}_{1}$ and $\mathrm{Vb}_{2}$ is evident by both polarity peaks precisely touching a reference line on the scope representing ground potential.

With the completion of this peak-to-peak to d-c voltage transfer adjustment, the electrostatic voltmeter is alternately connected from the a-c source. $\mathrm{V}_{1}$, to $\mathrm{V}_{\mathrm{dc}}$ which represents a known portion of the sum of $\mathrm{Vb}_{1}$ and $\mathrm{Vb}_{2}$. Any difference in the deflection of the electrostatic voltmeter for the two comparisons represents the ac-dc transfer error of the voltmeter. The error magnitude can be determined by readjustment of the $V_{\text {dc }}$ 
calibrated voltage divider, $R_{t}$, at the output of $\mathrm{Vb}_{1}$ and $\mathrm{Vb}_{2}$ for an ac-dc difference of zero at the voltmeter. The difference between the adjusted fraction of voltage at the voltage divider and $1 / 2 \sqrt{2}$ is the ac-de transfer error of the electrostatic voltmeter. The $2 \sqrt{2}$ term comes from having initially set each of the two dc voltages to the peak value of the sine wave. Test results at $50 \mathrm{~Hz}$ indicated an agreement between the peak-topeak comparator and the rms electrostatic voltmeter with an average precision of better than $0.003 \%$, and at no time did the data show a transfer error of greater than $0.01 \%$.

While Smith and Clothier had demonstrated the potentiality for precision a-c measurement with instrumentation of a basic electronic nature, a number of years went by before their ideas were expanded and development of instruments for commercial distribution became a reality.

\subsection{A Resistance Summing Peak ac-dc Voltage Comparator}

Recognizing the increasing need for precise a-c measurement in both the military field and in the standards laboratory, industry has responded within the last five years with a remarkable number and variety of precision instruments. These include highly stable $d-c$ power supplies, very low distortion a-c supplies, and a good many measuring devices. Among the latter is a peak ac-dc voltage comparator developed by P. Richman (7), for use over a frequency range of $50 \mathrm{~Hz}$ to $20 \mathrm{kHz}$. The basic circuit is shown in Figure 3. In the interest of versatility and wide frequency response the design avoids the use of a time-selection approach for examining the peak of each sine wave. Instead, it uses an ac-dc resistancesumming network with amplitude limiters which allow the viewing of a narrow slice of amplitude instead of time. As with the Smith-Clothier system, an oscilloscope serves as the null indicator.

Considering the circuit of Figure 3 in more detail, the selected polarity peak of the sine wave is shifted, with the aid of an identical pair of summing resistors, to a common reference by a d-c voltage of the opposite polarity. A back-to-back pair of silicon diodes connected at the summing junction clips off the signal excursion at approximately the 0.7 volt level in both polarity directions. The relay in the circuit switches at some uniform rate between the clipped sine wave peaks at the summing junction and the common reference line, which may be ground. After further amplification the waveform is again amplitude limited before application to an oscilloscope. The waveform at the scope face consists of a group of sine wave peaks appearing as sharp spikes in sequence with the zero reference line. A null balance is achieved by varying the $d-c$ source in such a manner as to bring the sharp peaks to the same reference leve1. In this way a peak ac-dc transfer has been made. By reversing the $d-c$ source polarity the other a-c peak can be compared, and averaging the two $d-c$ values will remove the effect of even-order harmonic distortion that may exist in the a-c source. This author suggests a variation in scope presentation. According to usual instructions the scope sweep is 
synchronized with the relay at the line frequency, $60 \mathrm{~Hz}$, producing the sequential pattern of spikes and reference line described previously. However, if the scope is synchronized from the a-c source, the sweep rate can be increased to the point where one expanded peak of the sine wave tip appears superimposed on the zero reference line. The advantage of this presentation is that there appears to be less confusion about setting the sine peak and the reference line into correspondence with ever present noise on both functions. Of added importance is the fact that the expanded function allows a detailed study of the waveform which may be influenced by noise and other disturbances. When used with near ideal a-c and $d-c$ sources, the described peak comparator can give consistent results with a precision of $0.005 \%$ over a wide frequency range.

\section{Description of the NBS Peak ac-dc Transfer Standard}

With an a-c source having known values of harmonic contamination, it is possible to calibrate a peak a-c to d-c instrument using existing rms thermal transfer standards. However, it would be more realistic and time saving to perform such a calibration using a laboratory peak transfer standard.

The basic idea in mind in the development of a new peak comparator was the search for a circuit that would offer a simple, direct comparison of the peak of a sine wave and a d-c source. It was expected that the desired simplicity would place some restrictions on operational flexibility, but the ultimate need was for a laboratory standard and not a field instrument.

In the design of the instrument the use of precision summing resistors for the $a-c$ and $d-c$ sources has been avoided; rather, the $a-c$ and $d-c$ voltages are time sequentially compared to a common reference. Eliminating the summing resistors removed a possible source of error that would have been present with unknown variations in their relative values. Also, with a resistive network there is the possibility of an ac-de error due to reactive components both within the resistors and external to them.

The system uses a pair of synchronized relays to slice out a time section of the sine wave at its peak in the manner of the Smith and Clothier scheme. These relays have time-overlapping closure, and are synchronized from binary frequency dividers and phase shifters. They extend the range of measurement to several thousand $\mathrm{Hz}$. The electronic amplifier was designed for simplicity. All possible storage elements, such as capacitors, have been deleted to give fast recovery and to minimize the frequency sensitivity of the circuit. External feedback loops with their usual reactive components have been avoided in order to achieve a flat frequency response with a near natural upper frequency roll-off characteristic.

Figure 4 is a block diagram showing the fundamental operation of the NBS instrument and a graph of the overlap operation of the relays is 
included in Figure 5. The overlap operation simulates a single-pole double-throw switching unit which samples the a-c for less than 100 microseconds, a requirement which no single relay could meet in synchronized operation. Selection of relay design was made on the basis of several requirements unique to this circuit. The chosen relays would have to be of the high speed type, that is reliable operation in excess of 100 cycles per second. Relays capable of high speed operation would maintain better synchronism and also require fewer frequency divider stages when operating with a $\mathrm{c}$ input frequencies of several thousand hertz. Relay designs with minimum contact bounce should be given preferential consideration. Long operating life is also important; the devices should function without degraded operation for millions of operating cycles. In the interest of stability at high speed operation, a relay excitation coil with low winding inductance and resistance would be easier to fit into a circuit design. The type of relay that best met the requirements was the commercially available reed relay with mercury-wetted contacts. They can be obtained for speeds of up to 200 operations per second, with less than $50 \mathrm{ohm}$ windings, 50 milliwatt operating power, and an operating life of more than 10 cycles. In addition, non-bridging contacts could be specified in order to avoid a common electrical path in the switching circuit between the $a-c$ and $d-c$ sources.

\subsection{Relay Synchronizer}

Figure 6 is a schematic diagram of the transistor circuit necessary to synchronize the relay operation with respect to the a-c source. The circuit can best be understood by following the signal flow from input to output with a brief explanation of the function of each electronic stage.

The input of the synchronizer system is the same a-c voltage that is to be compared with the d-c voltage. Silicon diodes are used to clip both polarities of the sine wave leaving a 0.7 volt square-wave-like signal at the input of an emitter follower, Q101. A Schmitt trigger circuit made up of Q102 and Q103 and designed with triggering levels close to zero reference is able to generate a large square wave output with rise and fall excursions that are close to the zero crossings of the input sine wave. Q104 is a phase-splitter stage with two outputs-one in phase at the emitter and the other output at the collector having a 180 degree phase relationship with the input. In a peak to d-c comparator it is desirable to examine both polarity peaks of the sine wave. In the system being described, a relay under the control of the d-c source polarity is used to select the proper phase output of Q104 for subsequent synchronizing purposes.

The instrument was designed to operate over a frequency range from 50 to $2400 \mathrm{~Hz}$. Since electro-mechanical relays cannot function at the higher speeds, six binary stages have been incorporated in the circuit for the purpose of frequency dividing the a-c input. A seven-position switch allows the selection from 1:1 to 64:1 frequency division; the 
selected position is chosen for a relay operating speed of about 50 operations per second. For example, in the case of an input a-c signal of $2400 \mathrm{~Hz}$, one would select the 64:1 division position. In this mode every 64 th peak of the $a-c$ source would be examined by the narrow time gate. No information is lost by this procedure; but the oscilloscope must have sufficient trace intensity to display an expanded pattern with a duty cycle that may be less than 0.1\%. Referring to Figure 6, the differentiated output of the binary selector switch is directed to emitter follower, Q117, which in turn operates two single-shot multivibrator stages, Q119, Q120, Q121, and Q122. Each multivibrator stage is used to generate a controllable time delay. At this point the system branches off into two independent channels for operating relay $A$ and relay $B$. Since the channels are identical, only one need be discussed. The time delay in single shot multivibrator, Q119 and Q120 is under the manual control of a front panel 10 turn precision potentiometer with digital dial for logging purposes. Both time delay multivibrators are initiated at the same time but with independent turn off times set by the precision potentiometers. The delay circuit serves two purposes - one function is to control operation of the relay in order to center the generated time gate exactly at the sine peak, and secondly, to stagger the two delay times in order to form a sampling time gate of the desired width as shown in Figure 5. Iogging of the digital dial settings helps in setting up the instrument for each new change of input frequency. The differentiated output of Q120 and the output of the reset signal handiing stage Q118 are used to trigger the binary stage Q123 and Q124 which is used to generate the waveform for subsequent excitation of the relay coll. Q127 is the output stage for driving the power amplifier.

The relay drive circuit is shown in Figure 7. Q201 is a power amplifier for the signal output of Q127 in the previous schematic diagram, Figure 6. Coupling from the last stage, Q201, to the relay coil is made through a capacitor, $\mathrm{C2O1}$, for the purpose of shifting the normally negative going voltage at the collector into a potential that swings both positive and negative with respect to common. This is done in order to improve the on-off operation of the single-side stable relays. The series resistor between capacitor and relay coil was selected on the basis of reliable operation with minimum current in the interest of high speed synchronization. As shown in Figure 5, the norma11y "OFF" side of the relay switching was chosen as the common "ON" position for the overlap operation. In this mode less contact noise was observed. The combined operation of the two relays acts as a single-pole double-throw switch that makes a synchronized closure on the a-c side for a time as short as 50 microseconds and a much longer closure time on the d-c side. Relay instability or "jitter" has been less than 10 microseconds in practice.

Since the instrument sequentially samples the a-c peak and the $d-c$ source, the polarity of the peak must correspond to the $d-c$ polarity. Some arrangement had to be designed into the system for translating the chosen 10-volt signal level down to the zero-state common reference. An isolated, stable, well filtered 10-volt supply is located between the 
relay output and the amplifier input. To simplify operating procedure, a polarity sensing circuit has been included which consists of stage Q203 and a pair of common variety relays $\mathrm{Rb} 3$ and $\mathrm{Rb} 4$. The transistor senses the $d-c$ source polarity and operates the relays in an appropriate manner. One relay establishes the polarity of the 10-volt series supply, while the other selects the proper phase polarity at Q104 in Figure 6 in such a manner that the delay circuits will position the time gate on the desired polarity peak.

\subsection{Amplifier}

The purpose of the amplifier is to provide the proper transfer circuit between the output of signal-gating relays and the oscilloscope input. A first requirement in considering a cholce of design is the degree of amplification itself. For oscilloscopes having a vertical deflection sensitivity between 0.01 and 0.1 volts per $\mathrm{cm}$, the instrument amplifier should have an amplification figure of from 10 to 100. Loading effects of the amplifier input on the $a-c$ and $d-c$ sources had to be considered, since the degree of measuring precision is to be close to $10 \mathrm{ppm}$ $(0.001 \%)$. Keeping the loading error below this figure implies an amplifier input impedance of at least 100,000 times the impedances of the two sources. Although modern precision $a-c$ and $d-c$ supplies may have source impedances of much less than $0.1 \mathrm{ohm}$, the amplifier was designed with an input impedance greater than 100,000 ohms.

Another aspect of the amplifier characteristic is the question of bandwidth. Since the a-c input frequency extends from 50 to $2400 \mathrm{~Hz}$, at first glance one might be tempted to assign a modest frequency response requirement to the amplifier. In order to avoid an amplitude correction factor greater than 5 ppms, the bandwidth of the circuit must be such that the upper corner frequency is at least 300 times the highest signal frequency involved. A bandwidth in excess of $1 \mathrm{MHz}$ was assigned as a requirement in the amplifier under discussion.

It can be assumed that low frequency roll-off is as pertinent to signal amplitude integrity as the high frequency case. This problem was circumvented by designing the amplifier to have d-c response. A very low frequency corner, by the use of large coupling time constants, would satisfy the bandpass requirements but would result in a bouncy pattern at the oscilloscope, due to the base line shift with varying signal amplitude. While on the subject of $d-c$ coupling some provision is required for adjusting the average level of the amplifier output, i.e., the amplifier should have a low $d-c$ offset between input and output levels. This is a circuit bias design problem.

Biasing of the first stage in the amplifier takes on more significance with the present application than would be usual for normal operations. The first stage should be biased in a manner that would leave the input terminal close to zero potential with respect to common reference. Ordinary solid-state stages may have quiescent input potentials of a volt or 
more due to biasing. Since the signal selecting relays are of the breakbefore-make type, there are two periods in each cycle of operation when the amplifier input is not connected to either signal source; if the quiescent input potential were other than near zero, the effect would be manifested as abrupt voltage transients at the amplifier output.

Figure 8 is the schematic diagram of the amplifier designed for use in the NBS transfer instrument. It represents a simple circuit that meets all of the stated requirements. The price paid for the simplicity has been a compromise of amplification stability and quiescent $d-c$ output level stability, neither of which affects the instrument accuracy.

The first transistor, Q301, presents an impedance of more than 100,000 ohms to the signal source while providing an ideal low impedance output for driving the following amplifying stage. Q301 is biased in a manner that allows signal excursions in both polarity directions, and also has a quiescent input potential of less than 35 millivolts. The second transistor, Q302, is the voltage amplifying stage with an amplification figure of between 10 and 20 from $d-c$ to about $1.5 \mathrm{MHz}$. A pair of transistors, Q303 and Q304, are used to provide an amplifier output impedance of less than 100 ohms; they also offer a symmetrical dynamic signal excursion range with minimum loading to the amplifying stage. In order to avoid the possibility of overloading the plug-in amplifier at the oscilloscope (many of which are now transistorized), a pair of germanium diodes have been placed at the amplifier output to limit signal excursions to less than one-half a volt.

\subsection{Power Supplies}

The word "ground" is conspicuously absent in the text of this report; in its place the word "common" has been substituted. In the various schematic diagrams the ordinarily used ground symbol has been replaced by a new symbol resembling a large "C", for common. Instead of using the instrument chassis as a common reference or ground, as is usually done, this instrument has a common reference that is electrically isolated from the chassis. The only connection to the chassis is the grounding wire in the 3-terminal power line plug. Two purposes are served - one is for safety from electrical shock, and the other is to allow the chassis and cabinet to act as a grounded electrical shield. Electrically isolating the circuitry from the chassis helps considerably to reduce the problem of interfering signal currents in ground loops. This problem is sometimes called "common mode effect". The problem has become more acute with the advent of the 3 wire power plug. If the instrument has one side of its signal lead grounded (as is the usual case), an interconnection of instruments may introduce unwanted ground currents into the signal circuit. Ironically, some manufacturers recommend plugging the 3 wire power plug into an adapter with a 2 prong output, in this way frustrating the original intent of the 3 wire plug. The indiscriminate use of ordinary coaxial connectors for low-level signals has led to much difficulty from ground loops. The braided shield of the cable carries ground currents as well 
as signal currents because its connectors make conductive contact with the chassis.

Additional paths for interference in many instruments are found within the power supplies, especially at the power transformer. Capacitive coupling from primary to secondary windings can result in noise being transferred from power line into electronic circuitry, despite the rectifier filters. To minimize this problem the three power supplies in the described instrument have been selected with very low capacity between windings and from windings to case. Figure 9 shows additional circuits that are required to adapt the power supplies to the needs of the system.

\section{Test Results}

The following tests were made in the Electrical Instrument Section of the Electricity Division. A thermal ac-de voltage comparator with an ac-dc transfer error of $10 \mathrm{ppm}$ or less was made available and was used as the standard in the following intercomparisons.

Figures $10 \mathrm{~A}$ and $10 \mathrm{~B}$ show how the instruments were connected. Both the $a-c$ and $d-c$ supplies are high precision instruments with amplitude stability of better than $25 \mathrm{ppm}$ for extended periods of operation; their noise content and the harmonic content of the a-c unit were less than $50 \mathrm{ppm}$.

Table 1 lists the results of an intercomparison between the NBS Peak ac-dc Voltage Comparator and a resistance summing peak comparator. For this group of tests the a-c supply was set for a fixed value of 10 volts peak amplitude. By means of oscilloscope observation of each comparator's output, the amplitude level of the $d-c$ supply was adjusted to match the peak a-c voltage. The 6 dial reading at the d-c supply was recorded for each determination. Results in the table show the difference in d-c dial readings in parts per mlllion (ppm) between sequential comparisons of the two peak comparators for each frequency. A positive sign indicates a higher $d-c$ reading by the NBS comparator over the other for the same $a-c$ value. Results shown cover 4 different a-c frequencies. Each determination is an average of the readings for both polarity peaks of the a-c sine wave. This procedure nearly eliminates the peak error effect due to evenorder harmonics present in the a-c voltage.

Note that a.1 the difference readings in Table 1 are in the same direction. In all cases the resistance summing peak comparator assigned a lower $d-c$ value to the $a-c$ peak than did the NBS peak comparator. The discrepancy was established by additional intercomparisons involving the thermal transfer standard, and will be described in the following paragraphs. One further remark on the results listed in Table 1 is the fact that no one test value departed by more than $20 \mathrm{ppm}$ for each frequency from the averaged values; most were less than $10 \mathrm{ppm}$. 
The results of the previous tests demonstrated the precision or repeatability of the peak comparators but no conclusions could be drawn as to their absolute ac-dc transfer accuracy. By comparing each peak comparator with the thermal ac-dc standard instrument, the ac-dc error of the peak comparators could be found. In addition, by taking the difference of the ac-dc errors of the peak comparators as judged by the thermal standard and comparing it with the difference readings described in Table 1 , a cross check of all measuring instruments will have been completed.

The second and third group of tests concerned the comparison of the peak comparators and the thermal transfer standard. Since these tests involve measurement of both the peak value and rms value of the a-c voltage, the need for waveform purity is apparent. During these tests the a-c output was kept at a constant 10 volts, rms. A $\sqrt{2}$ ratio transformer was included in the test circuit in order to normalize the 10 volt values between the peak reading instrument and the rms thermal instrument. The peak a-c value was compared with the voltage of a calibrated $d-c$ supply, using the peak-reading instrument in conjunction with an oscilloscope. The 6 dial reading of the $d-c$ supply was recorded. The same $d-c$ and a-c values were next compared by means of thermal transfer standard, and any difference between the two voltages was read at a calibrated galvanometer at the output of the thermal instrument. Recorded results in Tables 2 and 3 show the difference in ppm between the a-c and $d-c$ values as interpreted by the NBS Thermal Standard for a group of four frequencies. Table 2 represents results with the NBS peak comparator while Table 3 is for the resistance summing peak comparator. A positive sign indicates a higher a-c value than the $d-c$ one as measured by the thermal instrument. As was the case with the first group of tests, the individual values in most cases departed less than $20 \mathrm{ppm}$ from the average value.

On the basis of the results shown in Table 2, the N3S Peak Comparator has an ac-dc transfer acouracy of better than $0.0015 \%$ (15 ppm) for al1 the oparating frequencies except $50 \mathrm{~Hz}$. The maximum error occurred at a frequency of $50 \mathrm{~Hz}$, the particular frequency at which the a-c supply and output transformer has the poorest waveform purity.

Since the intended purpose for the NBS Peak Comparator is the direct measurement of the ac-dc error in other peak comparators, an evaluation of the accuracy of the data in Table 1 is in order. Now treating the NBS Peak Comparator as a standard, the results in Table 1 show the magnitude and direction of the ac-dc error for the resistance summing peak comparator. From Tables 2 and 3 the ac-dc difference between the two peak comparators can be judged as follows:

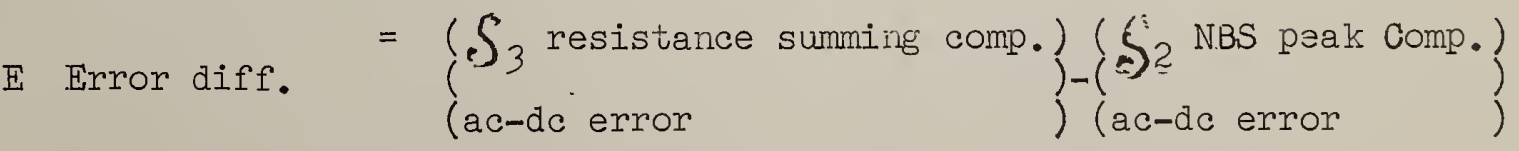


The ability of the NBS Peak Comparator to establish the ac-dc error in another peak comparator is shown in Table 4. In this Table the ac-dc error of the resistance summing comparator judged by the NBS Peak Comparator is compared with the error established by the NBS Thermal Standard. The agreement of the ac-dc error is better than $10 \mathrm{ppm}$ at all four frequencies.

The author wishes to thank Dr. Don Flach of the Electrical Instruments Section. Many hours of exacting tests were undertaken in the Standards Laboratories of that section in order to evaluate the described instrument. The high order of precision of the comparison tests results from Mr. Flach's careful contribution to the program. The author also thanks Mr. F. I. Hermach for his continued interest in the work and for having originally proposed the problem. 


\section{TABLE 1}

$$
\text { FREQUENCY - Hz }
$$

\begin{tabular}{llll}
$50 \quad 400 \quad 1000 \quad 2400 \quad$ RUN \\
\hline
\end{tabular}

$d-c$

$+16+6+27+21+1$

Difference between

$+26+16+50+41 \quad 2$

peak comparators

$\begin{array}{lllll}+21+16 & +51+51 & 3\end{array}$

PPM

$+11+16+36+46 \quad 4$

\section{$\left.S_{1}\right) \quad$ Average}

PPM

$+19+14+41+40$

NBS peak comparator vs resistance summing peak comparator 


\section{TABIE 2}

\section{FREQUENCY - $\mathrm{Hz}$}

\begin{tabular}{lllll}
$50 \quad 400 \quad 1000 \quad 2400 \quad$ RUN \\
\hline
\end{tabular}

$a c-d c$

$\begin{array}{lllll}+26 & -2 & +6 & +2 & 1\end{array}$

Difference

$+46+3 \quad-12+16 \quad 2$

PPM

$\begin{array}{lllll}+34 & -16 & -18 & -23 & 3\end{array}$

$\begin{array}{lllll}+37 & -11 & -18 & -4 & 4\end{array}$

$\left(S_{2}\right) \quad$ Average

PPM

$\begin{array}{lllll}+36 & -7 & -11 & -2\end{array}$

NBS peak comparator vs NBS thermal standard. 


\section{TABLE 3}

FREQUENCY - $\mathrm{HZ}$

$\begin{array}{lllll}50 \quad 400 \quad 1000 \quad 2400 \quad \text { RUN } & \end{array}$

$a c-d c$

$\begin{array}{llll}+48+9 & +28+58 \quad 1\end{array}$

Difference

$\begin{array}{lllll}+51+12 & +37 & +37 & 2\end{array}$

$+46+16+22+25 \quad 3$

$+38+19+15+36 \quad 4$

\section{PPM}



$\left(S_{3}\right)$ Average

PPM

$+46+14+23+39$

Resistance summing peak comparator vs NBS thermal standard, 


\section{TABIE 4}

FREQUENCY - $\mathrm{Hz}$

$\underline{50 \quad 400 \quad 1000 \quad 2400}$
$S_{I} \underset{\text { PPM }}{\text { from TABLE }} 1$

$+19+14+41+40$

$-S_{3}-S_{2}$ from

TABLES 2 and 3 PPM

$+10+21+34+41$

$S_{1}-\left(S_{3}-S_{2}\right)$

$\begin{array}{llll}+9 & -7 & +7 & -1\end{array}$

Agreement of established error in the resistance summing peak comparator by the two methods. 


\section{LIST OF FIGURES}

1. Half-Wave Diode Peak Rectifier.

2. Smith and Clothier Peak to Peak ac-dc Transfer Standard

3. Resistance Summing Peak ac-dc Comparator

4. Block Diagram of NBS Peak ac-dc Comparator

5. Relay Operation of NBS Comparator

6. Relay Synchronizing Circuit

7. Relay Driver

8. Amplifier

9. Power Supplies

10. Test Circuits 



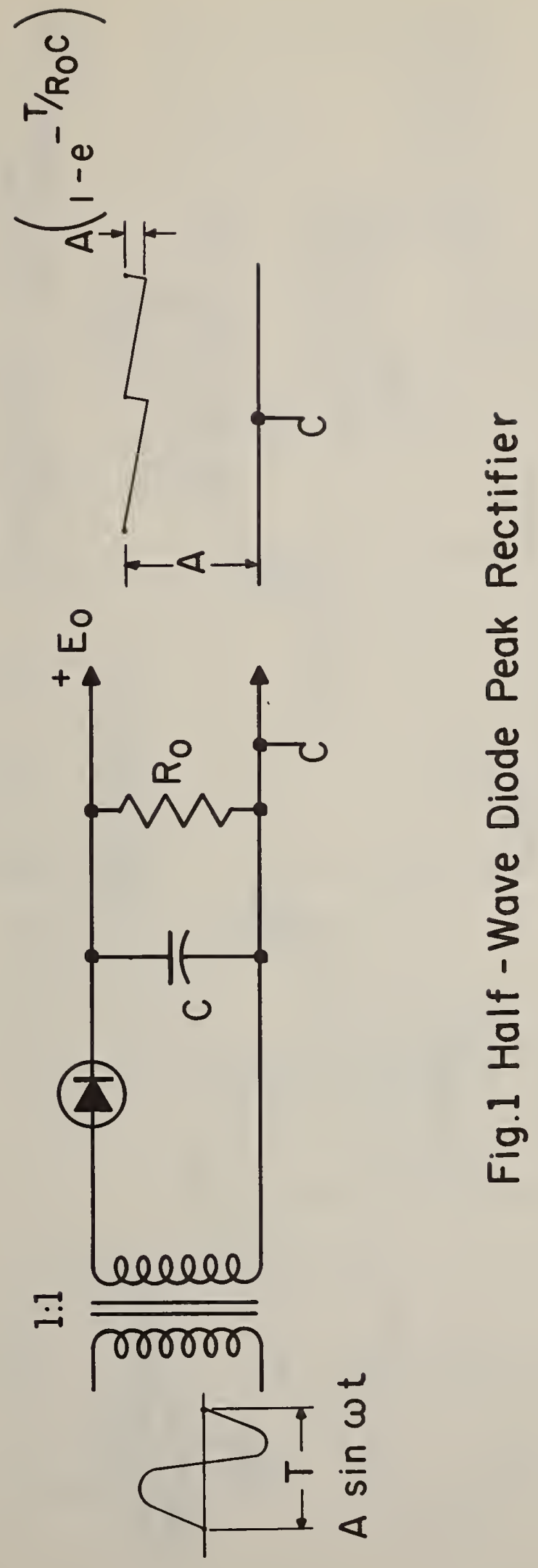




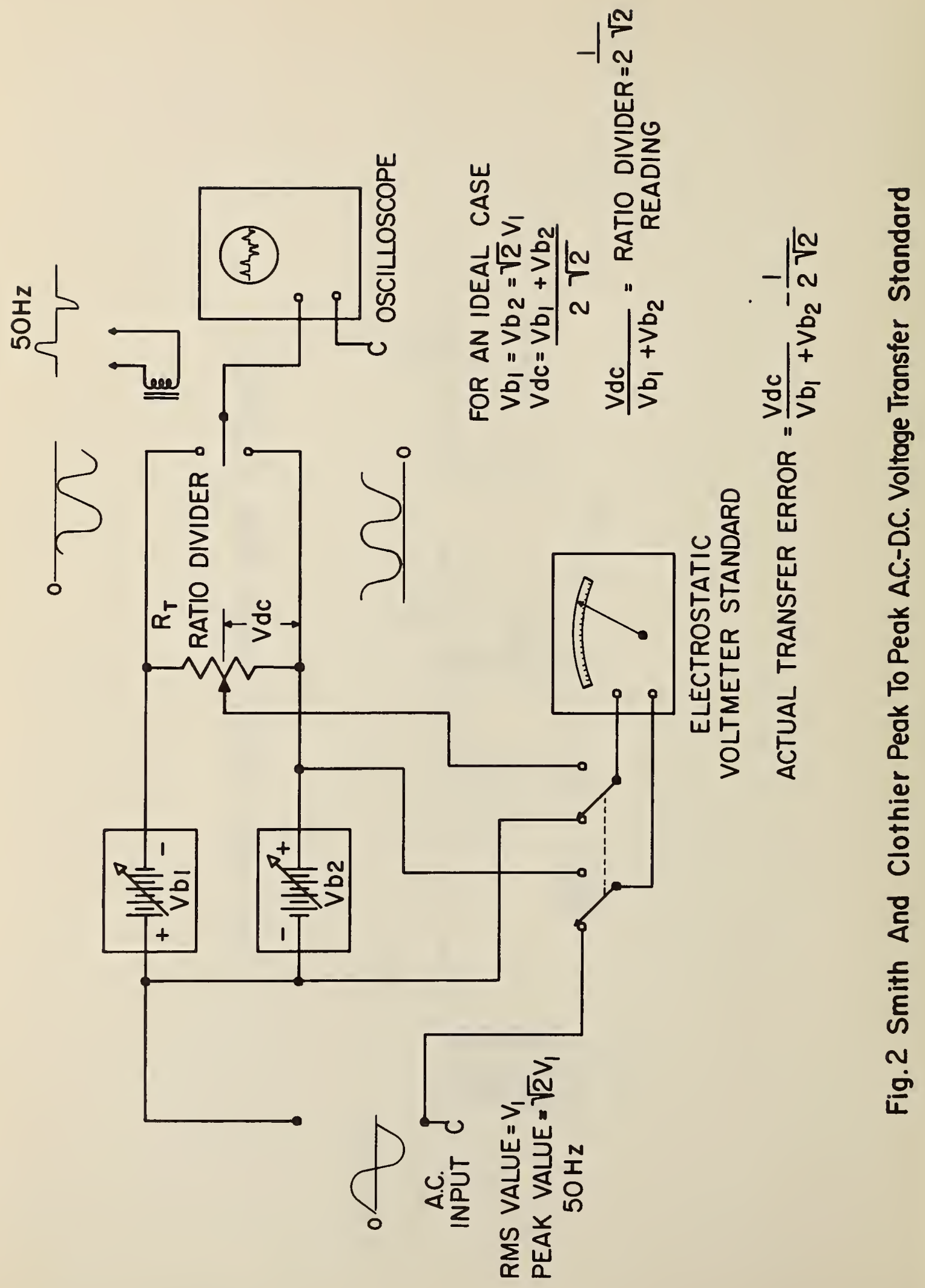




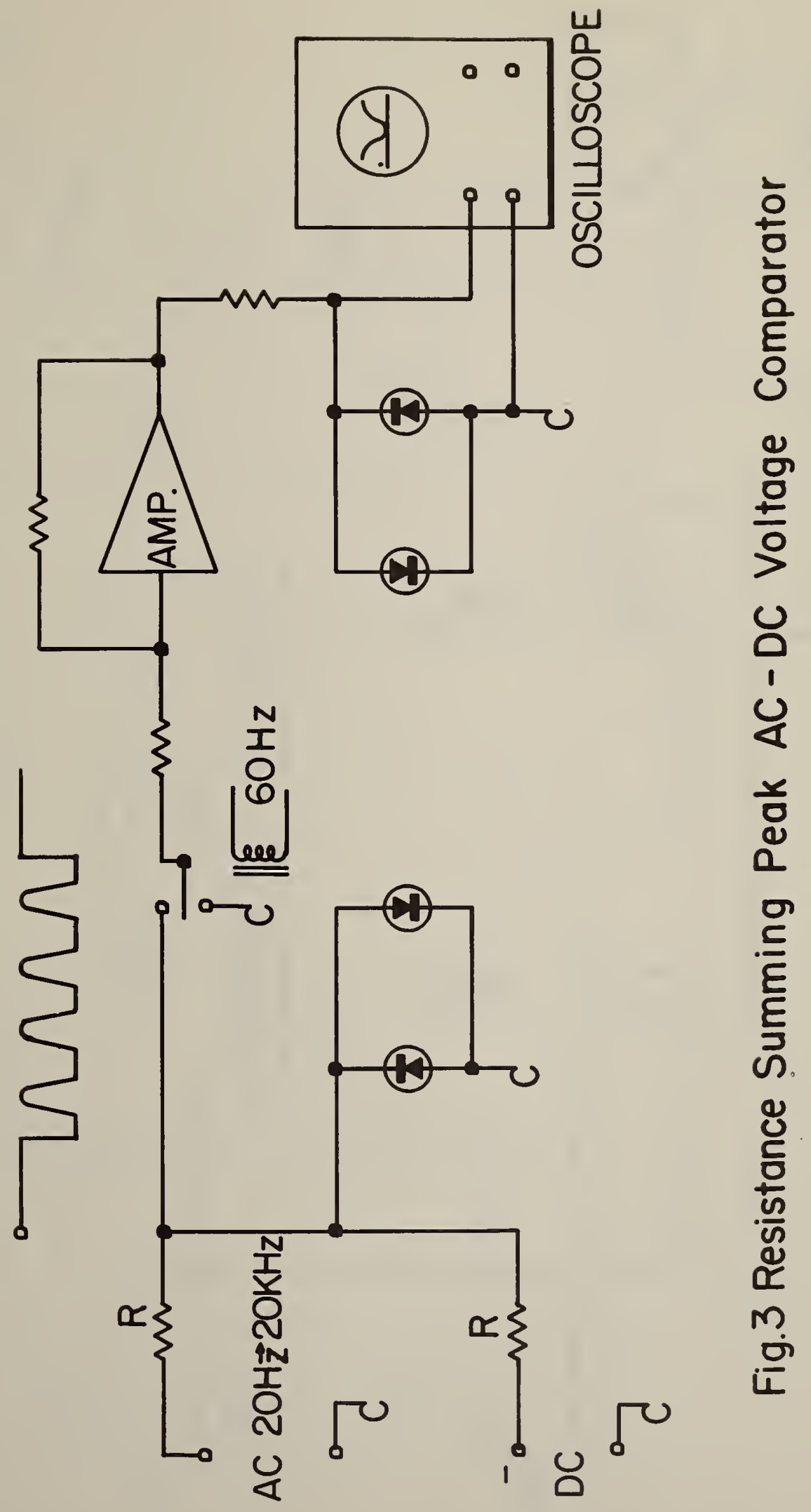


으
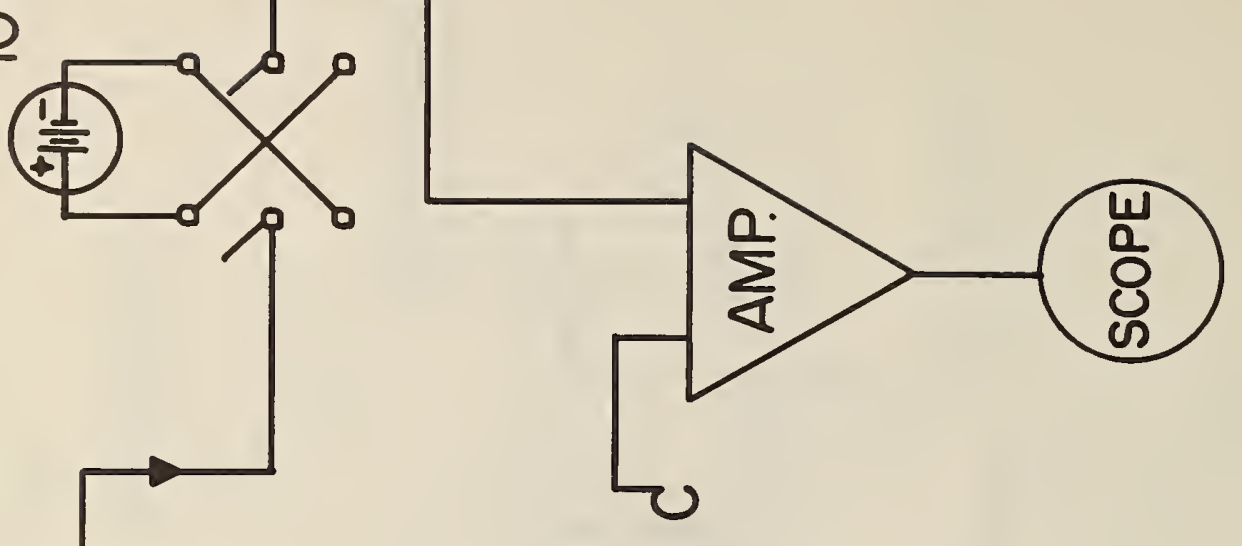

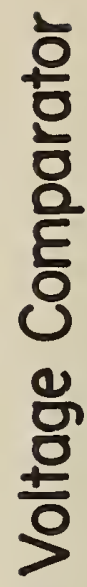




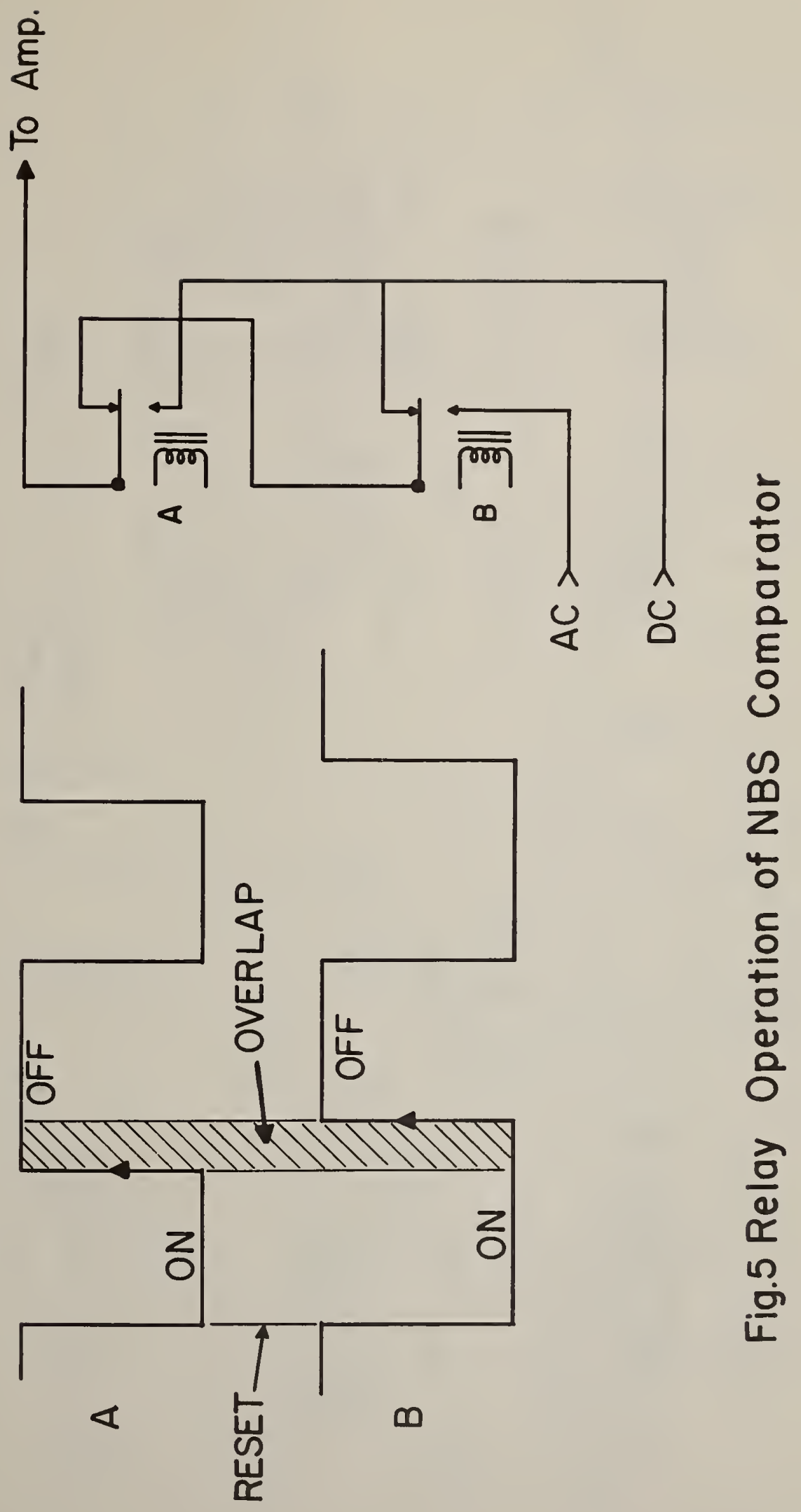



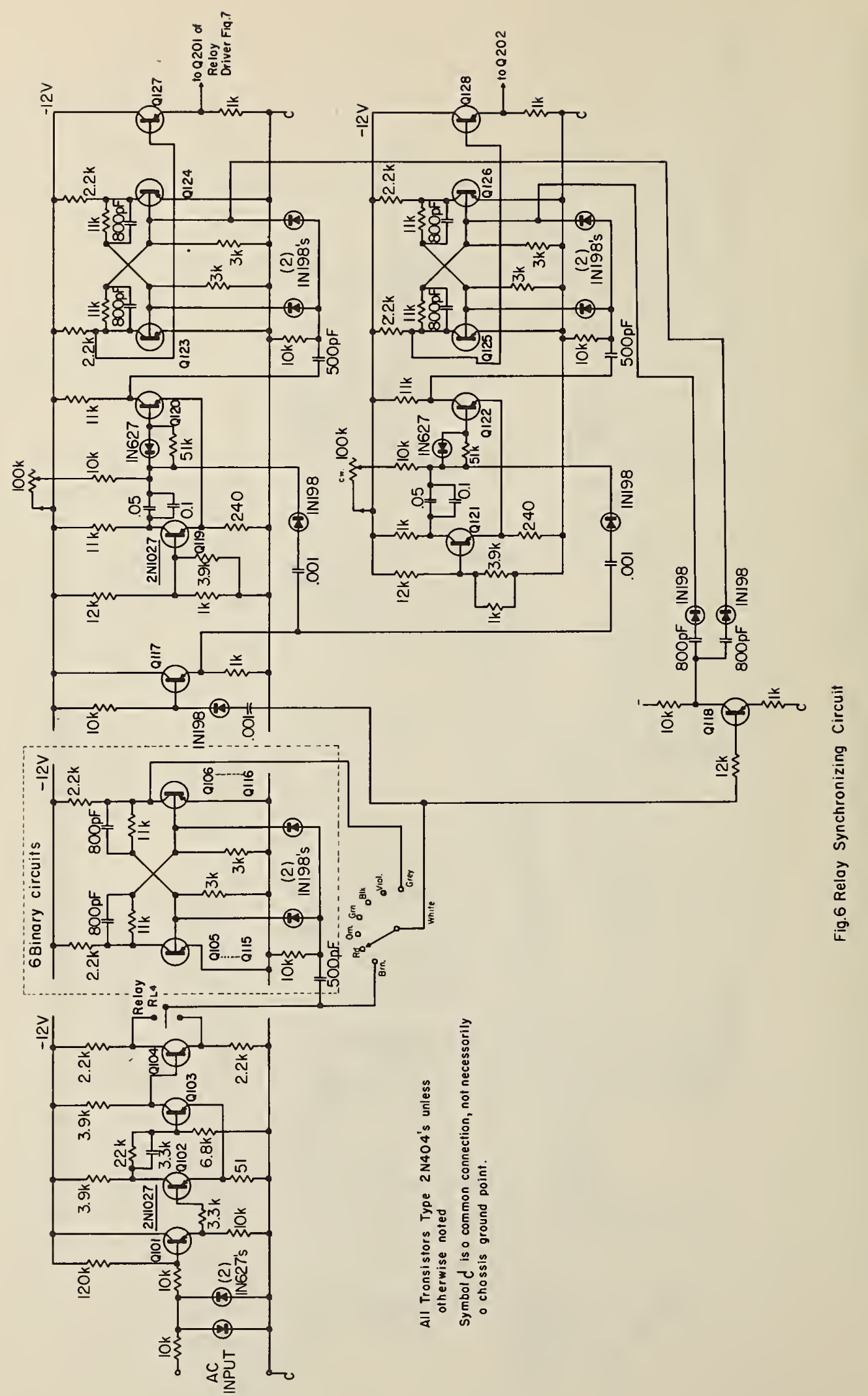


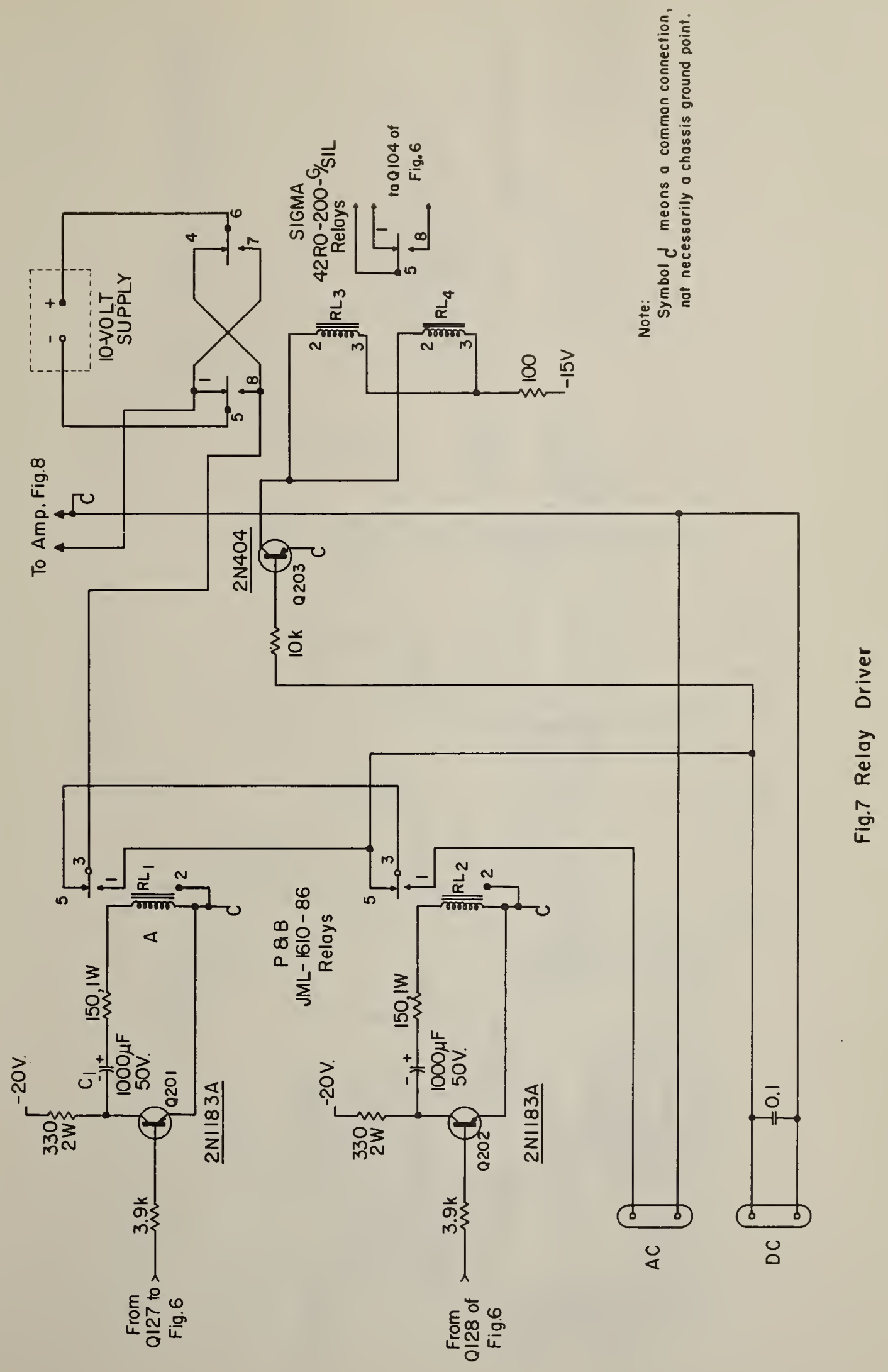




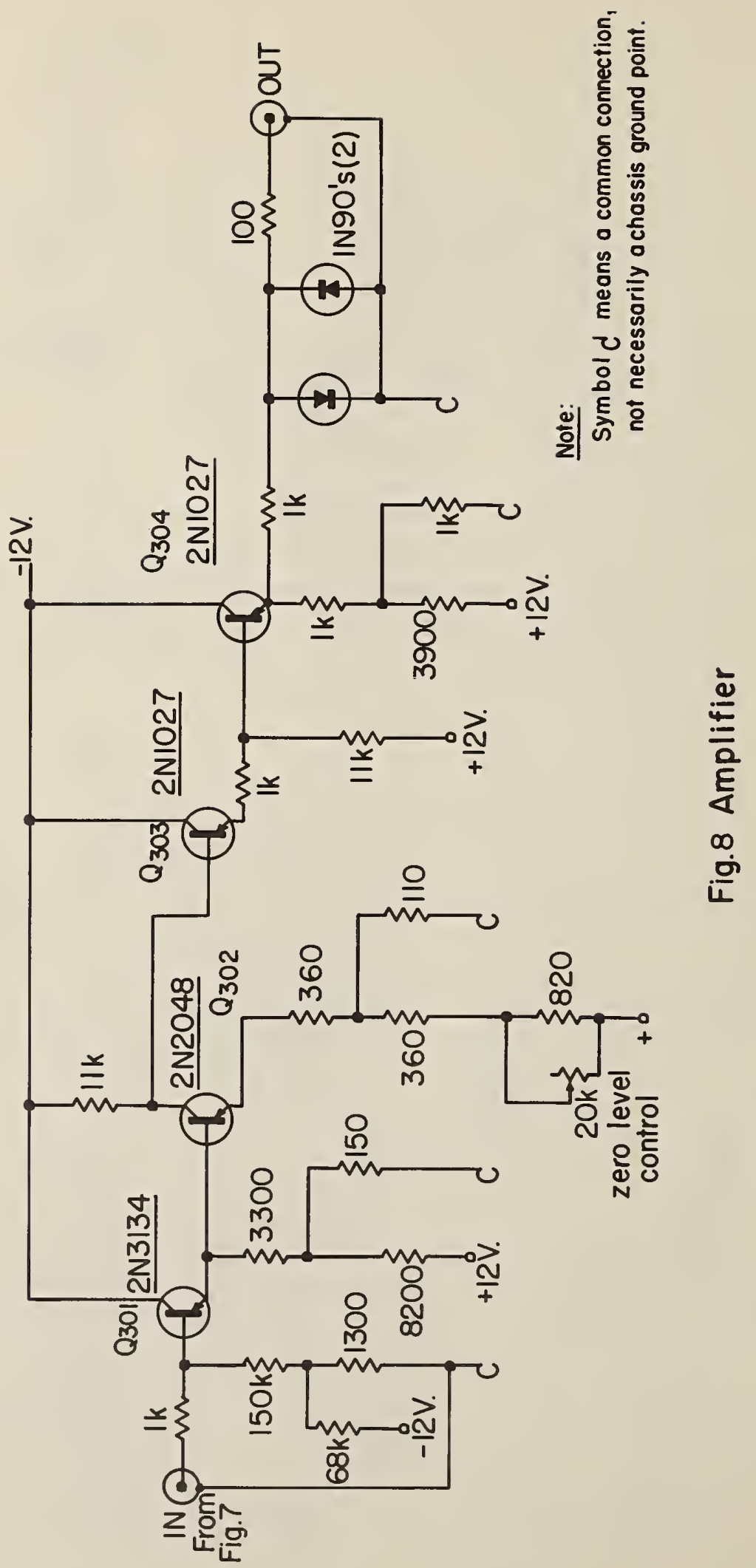



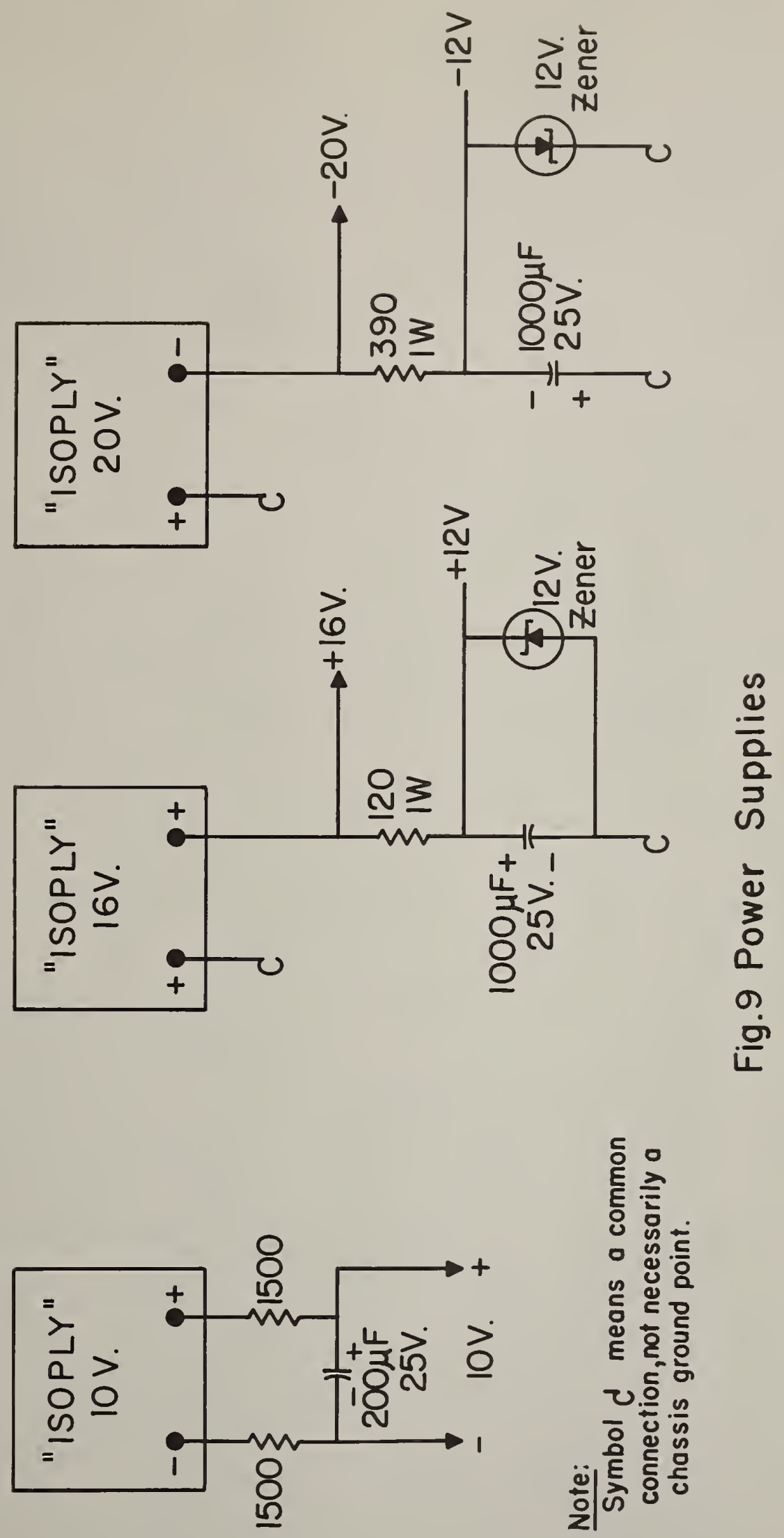

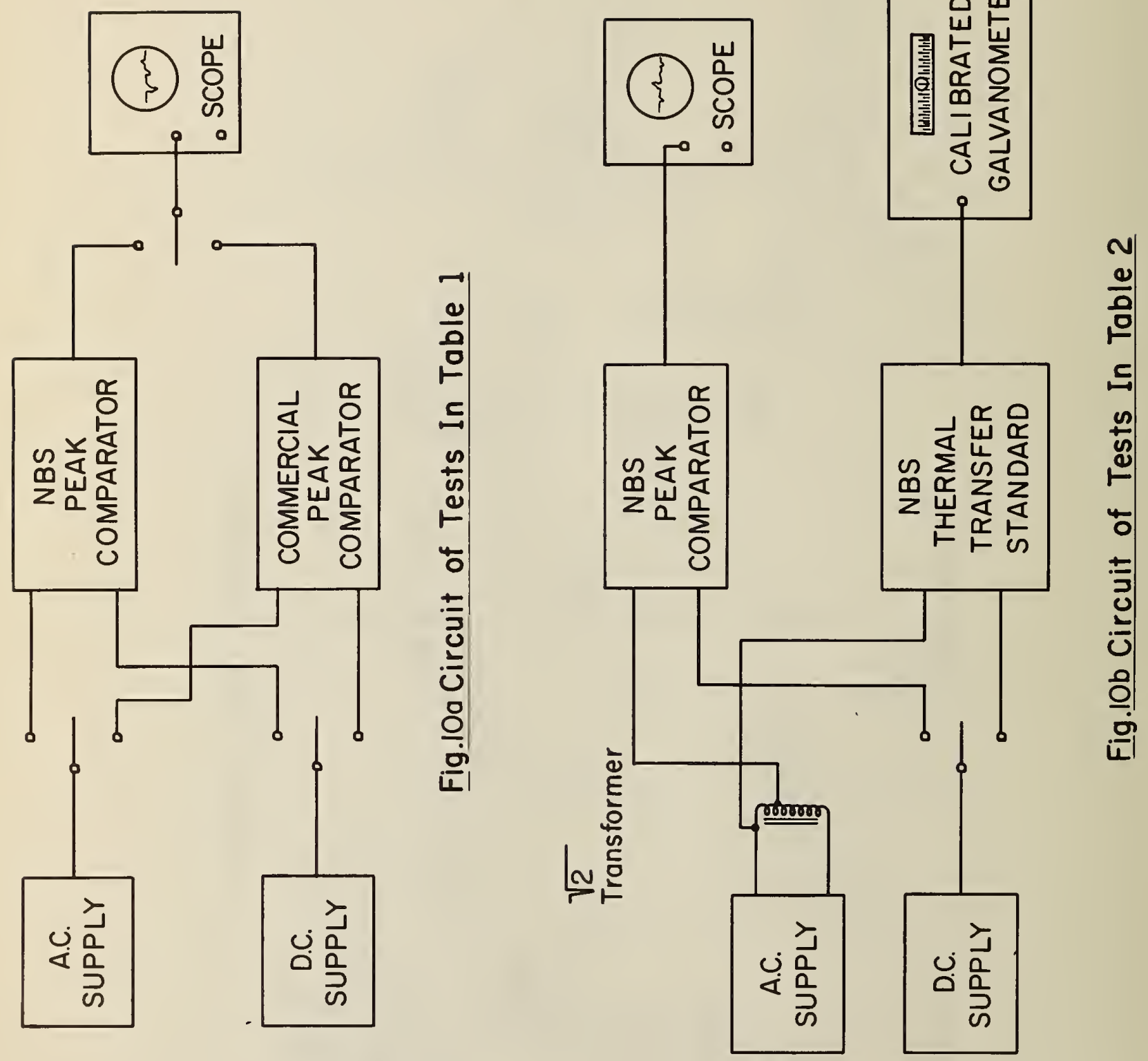

U.S. DEPARTMENT OF COMMERCE

WASHINGTON, D.C. 20230

OFFICIAL BUSINESS
POSTAGE ANO FEES PAID U:S. DFPARTMENT OF COMMERCE 